\title{
11.3
}

\section{Gastrine et Hélicobacter Pylori}

\section{B. MONGES}

Marseille

L'infection à Helicobacter Pylori ( H.P.) S'accompagne d'une hypergastrinémie basale et stimulée, d'une élévation du

Pepsinogène I et d'une diminution de I'acidité gastrique basale et stimulée (A $101 *$, A $181 *$ ). L'éradication de l'H.P. s'accompagne d'une correction de ces désordres (A $181 *$, A $143 *$ ).

Dans les séries d'ulcères duodénaux étudiés (A 140 *, A 181 *), l'infection à H.P. est retrouvée dans tous les cas. L'hypergastrinémie dans ces groupes est significativement supérieure à celle observée chez les sujets non ulcéreux infectés par H.P. (A $140 *$, A $181 *$ ). Il semble donc que la présence d'H.P. ne puisse expliquer à elle seule l'hypergastrinémie des sujets ulcéreux (A $140 *$ ).

Sur le plan histologique, l'infection à H.P. ne modifie pas le nombre de cellules à gastrine. L'augmentation de production de gastrine est donc vraisemblablement secondaire à un hyperfonctionnement induit directement ou indirectement par H.P. (A 75 *).

* Gastroenterology, 1991, 100, N $^{\circ} 5$, Part 2. 